\title{
A Case of Gastric Carcinoma with Breast Metastasis
}

\author{
Cansu KULUCAN ${ }^{1}$, Riza U. GURSU², Cüneyt MUDERRISOGLU ${ }^{1}$, \\ Nevra DURSUN ${ }^{3}$, Didem KARACETIN ${ }^{4}$ \\ ${ }^{1}$ Istanbul Training and Research Hospital, Department of Internal Medicine, Istanbul, TURKEY \\ ${ }^{2}$ Istanbul Training and Research Hospital, Department of Medical Oncology, Istanbul, TURKEY \\ ${ }^{3}$ Istanbul Training and Research Hospital, Department of Pathology, Istanbul, TURKEY \\ ${ }^{4}$ Istanbul Training and Research Hospital, Department of Radiation Oncology, Istanbul, TURKEY
}

\section{Dear Editor,}

Metastasis of solid organ tumors to breast is very rare. Metastases in the breast from extra-mammary sites are uncommon with the incidence ranging from $1,7 \%$ to $6,6 \%$ in autopsy series and 1,2 to $2 \%$ in clinical reports and being $2,7 \%$ in cytological series. The common causes of breast metastasis are hematological malignancies, malignant melanoma, lung, ovary, prostate, kidney carcinomas and carcinoid tumors. ${ }^{1,2}$ Gastric carcinoma metastasizing to breast is uncommon. Only $3 \%$ of solid organ tumors metastasizing to breast are gastric carcinomas. ${ }^{3}$ It is difficult to differentiate primary or metastatic tumors of the breast. Radiologic and immunohistochemical tests [steroid hormone receptors and gross cystic disease fluid protein (GCDFP-15)] are useful for diagnosis. ${ }^{4}$

A twenty-seven year old female patient was admitted with stomach ache and abdominal distention. On physical examination palpable masses on both breasts and ascites were found. Parasyntesis revealed malignant cytology. Computerized tomography showed diffuse thickness on stomach wall and multiple intra-abdominal lymphadenopathies. Gastroscopy revealed $2 \mathrm{~cm}$ ulceration in cardia. Bilateral mammographies of both breasts showed a 14x8 mm mass on right and 30x28 mm mass on left breast. Ultrasonography revealed multiple axillary lymphadenopathies. Biopsies from both breast masses and gastric ulcer was found to be poorly differentiated adenocarcino- ma (Figure 1). Immunohistochemical studies showed positivity for CK7, CK20 and, negative staining for PRand GCDFP-15 revealing diagnosis of primary gastric cancer with breast metastasis. Multiple bone metastases as well as multiple lymphadenopathies were also found by 18 -fluorodeoxyglucose positron emission tomography scan. Chemotherapy was not given due to poor performance status of patient and best supportive care was planned. Patient's condition worsened and she was dead a few days later from diagnosis.

Gastric cancer metastasis to the breast is rare with nearly 30 cases reported in the literature. The $91,7 \%$ of breast metastases from gastric carcinomas are female. Median survival after breast metastases was found to be 10 months. The $88,2 \%$ of breast metastasis had a history of malignancy, but no primary tumor was found at remaining. ${ }^{2}$

It is difficult to differentiate breast masses whether it is a primary tumor or a solid organ metastasis. Diagnosis usually can be made on routine frozen-section biopsy examination, although it may be necessary to perform detailed examination on the entire excisional biopsy specimen to give a correct diagnosis. Primary breast carcinoma must be the first choice for diagnosis in patients with solid mass on the breast. In patients having a history of malignancy, solid masses of breast must be considered firstly as metastasis. 


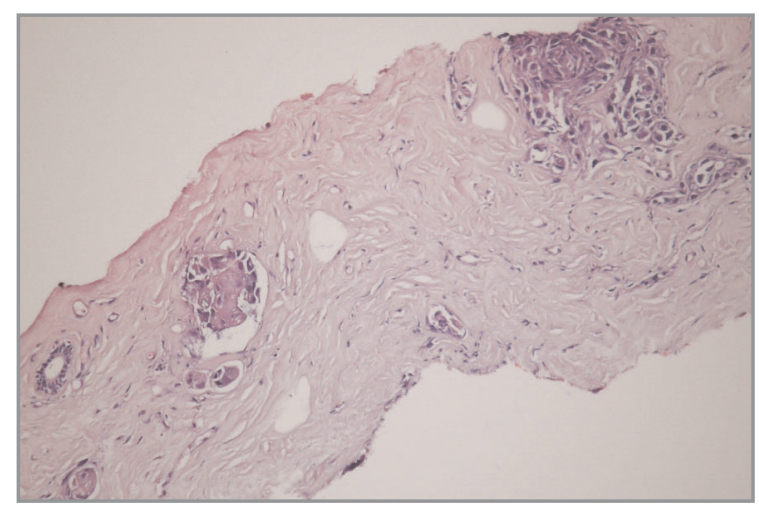

Figure 1. Metastasis of a poorly differentiated gastric adenocarcinoma in breast lymphatics and stroma (H\&E stain).

The intestinal pattern of gastric carcinoma may resemble invasive ductal carcinoma of the breast, and diffuse gastric carcinoma may resemble invasive lobular carcinoma of the breast. Columnar mucin-secreting cells favor gastrointestinal origin. Immunohistochemical studies are also helpful for differentiating the diagnosis. Studies that are helpful are ER, PR, GCDFP-15, CK20, and CDX2 ${ }^{8.9}$

In primary gastric carcinomas ER, PR, GCDFP-15, CK20 and CDX2 are found to be negative where as $\mathrm{CD} 20$ and CDX2 are negative in primary breast cancers. ${ }^{10}$ Some earlier reports describe ER-positive gastric carcinoma, but recent studies suggest this marker is particularly useful in the distinction from diffuse gastric carcinoma. Several recent studies did not find GCDFP-15-positive stomach cancer. CDX2 is present in between $20 \%$ and $70 \%$ of gastric carcinomas but not in breast cancer. Our patient had CK7 $(+)$, CK20 (+) and PR (-), GCDFP-15 (-) pointing out the diagnosis of primary gastric carcinoma. Computerized tomography and endoscopy are also helpful to diagnose primary gastric carcinomas.

Breast metastasis of gastric carcinoma is rare and with poor prognosis. It is very important to differentiate the diagnosis of a breast mass. Correct diagnosis leads to appropriate choice for treatment and save patients for unnecessary procedures.

\section{REFERENCES}

1. McCrea ES, Johnston C, Haney PJ. Metastases to the breast. Am J Roentgenol 4: 685-690, 1983.

2. Williams SA, Ehlers RA, Hunt KK, et al. Metastases to the breast from non-breast solid neoplasms: presentation and determinants of survival. Cancer 110: 731737, 2007.

3. Alva S, Shetty-Alva N. An update of tumor metastasis to the breast data. Arch Surg $134: 450,1999$.

4. Sato T, Muto I, Fushiki M, et al. Metastatic breast cancer from gastric and ovarian cancer, mimicking inflammatory breast cancer: report of two cases. Breast Cancer 15: 315-320, 2008.

5. Lee $\mathrm{AH}$. The histological diagnosis of metastases to the breast from extramammary malignancies $\mathrm{J}$ Clin Pathol 60: 1333-1341, 2007.

6. Quershi SS, Shrikhande SV, Tanuja S, Shukla PJ. Breast metastases of gastric signet ring cell carcinoma: a differential diagnosis with primary breast signet ring cell carcinoma. J Postgrad Med 51: 125-127, 2005

7. Boutis AL, Andreadis C, Patakiouta F, Mouratidou D. Gastric signet-ring adenocarcinoma presenting with breast metastasis. World J Gastroenterol 12: 295861, 2006.

8. Kwak JY, Kim EK, On KK. Radiologic findings of metastatic signet ring cell carcinoma to the breast from stomach. Yonsei Med J 41: 669-672, 2000.

9. Wick MR, Lillemoe TJ, Copland GT, et al. Gross cystic disease fluid protein-15 as a marker for breast cancer: immunohistochemical analysis of 690 human neoplasms and comparison with alpha-lactalbumin. Hum Pathol 20: 281-287, 1989.

10. O'Connell FP, Wang HH, Odze RD. Utility of immunohistochemistry in distinguishing primary adenocarcinomas from metastatic breast carcinomas in the gastrointestinal tract. Arch Pathol Lab Med 129: 338-347, 2005.

\section{Correspondence:}

Dr. Riza Umar GÜRSU

Topkapı Mahallesi

Pazartekkesi Sokak

Atakol Apt. No: 48/11

Topkapı, Fatih

ISTANBUL / TURKEY

Tel: (0212) 5884400 / 6222

e-mail: rumar80@yahoo.com 